\title{
Der Dieselskandal oder das Ethik-Problem moderner Unternehmensführung
}

\author{
Angesichts der Skandale bei Deutsche Bank, VW und anderen Automobilkon- \\ zernen scheint es in Deutschland mit der Managerethik nicht gut bestellt zu \\ sein. Unethische Entscheidungen fallen auch zu Lasten der Gesellschaft. \\ Woran liegt das? Einerseits besteht bei vielen Managern die Vorstellung, \\ dass sich ethisches Verhalten nicht auszahlt andererseits, werden auch die \\ falschen Manager befördert. Einem großen Teil fehlt die Persönlichkeit, um \\ Führungskraft zu sein. Hinzu kommt eine variable Vergütung, die nur den \\ kurzfristigen Erfolg belohnt.
}

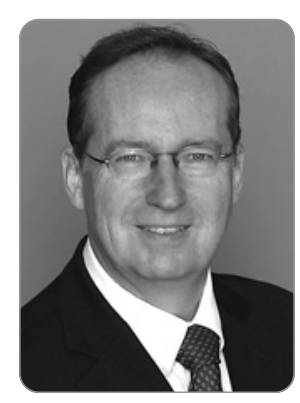

Prof. Dr. Christian A. Conrad

lehrt Volkswirtschaftslehre und Wirtschaftsethik an der Hochschule für Technik und Wirtschaft des Saarlandes. Er war vorher Senior Key Account Manager im nationalen und internationalen Unternehmenskundengeschäft einer deutschen Großbank. Er hat zahlreiche Bücher zum Thema Wirtschaftsethik veröffentlicht.

Stichwörter: Wirtschaftsethik, Unternehmensführung, Dieselskandal, variable Vergütung, ethische Personalauswahl

\section{Falsche Vergütungssysteme}

Das Beispiel von VW zeigt die Bedeutung der Managerethik für den langfristigen Unternehmenserfolg. Wie bei der Deutschen Bank wird deutlich, dass sich unethisches Verhalten nur kurzfristig aber nicht langfristig auszahlen kann. Ein risikofreudiges am kurzfristigen Erfolg orientiertes Verhalten des Managers kann durch falsche Anreize (Horal hazards) hervorgerufen werden. Solche Fehlanreize gehen von einer hohen erfolgsabhängigen Entlohnung aus, wenn sie kurzfristig ausgerichtet ist. Eine einseitige Beteiligung der Manager an der positiven Unternehmensentwicklung, also eine Gewinnbeteiligung ohne eine Verlustbeteiligung kann darüber hinaus zu einer starken Risikofreudigkeit des Managers auf Kosten der Unternehmenseigentümer führen. Wie experimentell gezeigt wurde, ist eine risikoadäquate, also ausgewogene variable Entlohnung wichtig, um Verzerrungen bei der Entscheidungsanreizen des Managers zu vermeiden (vgl. Conrad, 2015).

Dies war bei den von den Skandalen betroffenen Unternehmen nicht der Fall. Im Gegenteil, auch eine strafrechtliche Verfolgung der Manager findet in der Regel nicht statt. Teils liegt dies daran, dass nachträglich eine Mitverantwortung des Unternehmens festgestellt wird, teils kommt es aber auch zu einem außergerichtlichen Vergleich. Darüber hinaus schützen die Manager Haftpflichtversicherungen. Überwiegend kann man den Managern keinen Vorsatz nachweisen, weshalb das Unternehmen, genauer die Aktionäre für das ethische Fehlverhalten der Manager haften. Im Dieselskandal scheint zumindest die USA eine strafrechtliche Verfolgung einzelner Manager durchsetzen zu wollen. Das Fehlverhalten der Manager läßt sich somit auf die einseitigen Bonisysteme zurückführen, geht aber tiefer.

\section{Probleme der Corporate Governance}

Laut Stanford-Professor Jeffrey Pfeffer sind erfolgreiche Manger egoistisch, verlogen und rücksichtslos. Pfeffer stellt nicht in Frage, dass die Unternehmen von einem ethischen Führungsstil profitieren würden, er sieht dies aber als unrealistisch an und empfiehlt jungen Managern sich unethisch zu verhalten, um Karrieren zu machen. Um erfolgreich zu sein, muss man laut Pfeffer als Manager fies sein. Erfolgreiche Manager würden in ihrer eigenen Realität leben und sich und andere täuschen. Laut Pfeffer wirken die guten Eigenschaften, die Tugenden wie Bescheidenheit, Aufrichtigkeit und Vertrauen karrierehinderlich. Nur wer auffällt wird befördert. Deshalb würden Narzissten öf- 
ter Führungskräfte, weil ihr übersteigertes Selbstvertrauen von anderen Menschen fälschlicherweise als Kompetenz wahrgenommen wird. Lügen werden gemäß Pfeffer in den Unternehmen nicht sanktioniert und gehören zum Führungsalltag. Wer lügt insbesondere über seine eigenen Leistungen kommt deshalb weiter. Hierzu gehört auch das Brechen von Versprechen. Vertrauen ist so Pfeffer für die Unternehmen verzichtbar, weil Versprechen so oft ungestraft gebrochen werden. Pfeffers Thesen bzw. sein Buch "Leadership BS Fixing Workplaces and Careers One Truth at a Time" wurde 2015 Finalist bei der Financial Times und bei McKinsey Business Book des Jahres (vgl. Pfeffer, 2015, Schwertfeger, 2016).

Pfeffers Thesen über das unethische Verhalten der Manager werden von der langjährigen deutschen Psychotherapeutin und Personalberaterin Leitner bestätigt. Ihrer Meinung nach kursieren Psychospielchen vor allen in großen Firmen, weil dort oft keine Werte vorgelebt werden. Oben würden sich immer mehr Ego-Typen breitmachen, die nur an die eigene Karriere denken, denen das Wohl des Unternehmens gleichgültig sei (vgl. Dahlkamp, 2015).

\section{Ethische Unternehmensführung}

Haben Pfeiffer und Leitner recht? Zahlt sich ethisches Verhalten für die Führungskräfte und die Unternehmen nicht aus? Kann und muss man dagegen etwas tun?

Die Wirkungen von unethisches Verhalten der Vorgesetzten und die Wirkungen auf die Produktivität können auch am Beispiel des konkursgegangenen ehemaligen US-Vorzeigeunternehmens ENRON demonstriert werden. Behandelt das Unternehmen die Mitarbeiter sehr schlecht, wird die Loyalität aufgekündigt. Zuerst kommt die innere Kündigung und dann versuchen die Mitarbeiter, sich zu wehren bzw. die Nachteile zu kompensieren, indem sie gegen die Interessen des Unternehmens arbeiten. Das Ergebnis ist ein unproduktives Betriebsklima. Die Zugehörigkeit der Mitarbeiter zum Unternehmen bewirkt ein spezifisches Gruppenverhalten, das sich durch das Konzept der Unternehmenskultur beschreiben lässt. In Gruppen übernehmen die Menschen Rollen, die ihr Verhalten beeinflussen. Gruppen bewirken mit eigenen Normen ein gruppenangepasstes Moralverhalten. Eine unethische Unternehmenskultur ist ein Wettbewerbsfaktor, weil sie direkt die Motivation und die Zusammenarbeit der Mitarbeiter negativ beeinflusst. Die Mitarbeiter werden über die Unternehmenskultur sowie durch Vorbild, Belohnungen und Sanktionen der Führungskräfte sozialisiert. Für eine unethische Unternehmenskultur sind deshalb zu allererst die Führungskräfte verantwortlich (vgl. Conrad, 2016, S. 249 ff.).

Ein ethischer Führungsstil beschränkt sich nicht allein auf ein ethisches Verhalten, sondern muss als aktive ethi- sche Führung ethische Werte postulieren, als Vorbild vorleben, ethisches Verhalten belohnen und Verstöße sanktionieren. Empirische Untersuchungen zeigten, dass ethische Führung von den Mitarbeitern vor allem mit Fairness und Ehrlichkeit assoziiert wird. Es konnte gezeigt werden, dass ethische Führung bei den Mitarbeitern $\mathrm{zu}$ einer Bereitschaft führt, sich überdurchschnittlich im Job zu engagieren und Probleme an das Management zu berichten (vgl. Bass/Avolio, 2000, Brown/Treviño, 2006, S. 597). Langfristig bewirkt ein ethischer Führungsstil somit mehr Produktivität.

Es scheint in der Wirtschaft an der Kenntnis über die Bedeutung von ethischen Werten für den langfristigen Unternehmenserfolg zu fehlen. Gemäß einer Studie von $B u ß$ hält nur ein Drittel der deutschen Spitzenmanager ethisch verantwortliches Handeln nicht nur für wünschenswert, sondern setzt es auch weitgehend im Unternehmensalltag um. Etwa $13 \%$ der deutschen Spitzenmanager sind sogar der Auffassung, Wirtschaft und Moral seien letztlich unvereinbar (vgl. Buß, 2009, S. 11 ff.).

Es fehlt somit bei den Führungskräften an dem Grundverständnis, dass Mitarbeiter am besten durch das eigene positive Vorbild geführt werden. Eine Führungskraft muss vom untergebenen Mitarbeiter als Persönlichkeit akzeptiert werden (Personalautorität), wozu sie sich auch ethisch verhalten muss. Nur dann wird es der Führungskraft gelingen, das Vertrauen der Mitarbeiter zu gewinnen und sie da$\mathrm{zu}$ zu motivieren, gerne den Anweisungen $\mathrm{zu}$ folgen und sich für das Unternehmen einzusetzen. Ziel sollte eine positive Corporate Identity sein: Der Mitarbeiter geht gerne ins das Unternehmen und möchte es weiterbringen. Seine Arbeit gibt ihm Lebenssinn und Erfüllung.

Der Mitarbeiter erwartet einen moralischen Vorgesetzten nicht $\mathrm{zu}$ letzt $\mathrm{zu}$ seinem eigenen Vorteil. Er ist von ihm abhängig und möchte nicht von ihm geschädigt werden. Lehnt der Mitarbeiter die Führungskraft ab, wird er ihr nicht vertrauen, sie also auch nicht uneingeschränkt informieren. Vielmehr kostet es den Mitarbeiter viel Kraft, die Anweisungen eines Vorgesetzten zu befolgen, den er persönlich ablehnt. Dies erzeugt Agressionen, die sich auch gegen den Vorgesetzten richten können.

Es reicht somit nicht, ethische Verhaltenskodexe $\mathrm{zu}$ beschließen, sie müssen auch durch die Führungskräfte durchgesetzt und vorgelebt werden. Nicht nur die Leistung des Mitarbeiters, sondern auch sein Verhalten Dritten gegenüber muss kontrolliert werden.

Entsprechen aber die Führungskräfte diesen Anforderungen?

Eine Unzufriedenheit der Mitarbeiter mit den Führungskräften führt alleine schon durch Kündigungen $\mathrm{zu}$ einem Kosten- und Produktivitätsausfall. So zeigte 2014 eine Befragung der Beratungsfirma Information Factory von 2687 
Mitarbeitern und Führungskräften in der Schweiz, dass $62 \%$ der Befragten bereits einmal wegen eines Vorgesetzten gekündigt haben. Bei einer vergleichbaren Befragung in Deutschland im Jahr 2013 gaben dies $50 \%$ der Befragten an, wobei zusätzliche $20 \%$ angaben, sie standen kurz davor zu kündigen (vgl. Information Factory, 2014, S. 4). Untereinander charakterisieren sich Führungskräfte gemäß Schieffer häufig als egozentriert, selbstverliebt, eitel, macht- und statusversessen, distanziert und unkommunikativ (Vgl. Schieffer, 1998, S. 296 ff.). Somit scheint auch einem großen Teil der Manager die Persönlichkeit zu fehlen, um ein Unternehmen erfolgreich zu führen. Wieso sind sie dann an die Unternehmensspitze gekommen?

\section{Ethische Personalauswahl}

Windolf stellte bei seiner Untersuchung der Betrugsaffären Enron und WorldCom fest, dass hier die Unternehmens-Selektionsmechanismen für Führungskräfte dazu führten, dass die Personen ausgewählt wurden, die aggressiv und im Grenzfall auch mit krimineller Energie ein hohes Einkommen anstreben (vgl. Windolf, 2003, S. 195 f.). Prinzipiell sollten gemäß dem Ansatz der ethischen Personalauswahl nur Mitarbeiter eingestellt oder befördert werden, die den ethischen Kriterien des Unternehmens entsprechen (vgl. Conrad, 2016, S. 300 ff.). Hierauf wird gerade bei Führungskräften oft zu wenig wert gelegt. Gerade externe Besetzungen von Führungspositionen stellen hier ein Risiko dar. Nur bei internen Beförderungen sind die sozialen Kompetenzen bekannt. Das Unternehmen kennt den Mitarbeiter schon seit Jahren. Dieser hat sich verdient gemacht und versucht, sich hochzuarbeiten (vgl. Conrad, 2017). Leider gibt es auch bei internen Beförderungen oft Entscheidungsverzerrungen durch Parteinahme und Beziehungsgeflechte im Unternehmen. Dem könnte durch die Hinzuziehung externer Gremien (z. B. einer Ethikkommission) bei den Personalentscheidungen vorgebaut werden.

Die Werteorientierung von Mitarbeitern und Führungskräften lassen sich darüber hinaus durch Fragebögen, Fallstudienbearbeitung, Gruppendiskussionen und Planspiele ermitteln. Beispielsweise kann man in einer Gruppe ein ethisches Dilemma oder auch die wünschenswerten Eigenschaften von Führungskräften diskutieren lassen (vgl. Göbel, 2010, S. 235). Es wurden aber auch verschiedene Testverfahren speziell zur Erfassung der ethischen Orientierung und Urteilskraft von Führungskräften entwickelt (vgl. Staffelbach, 1994, S. 248).
Auch das Verhalten der Führungskräfte muss angesicht der vielen Hierarchiestufen gerade in Großunternehmen überwacht werden. Hier eignet sich insbesondere eine regelmäßige annonyme Evaluation der Führungskräfte durch die Mitarbeiter. Ferner sollten interne Ethikbeauftrage wie die interne Revision die Prozesse im Unternehmen auf die Einhaltung der Integritätsprogramme kontrollieren. Externe Ethikbeauftragte, die wie bspw. Anwälte zur Verschwiegenheit verpflichtet sind können für den Fall zur Verfügung stehen, dass Mitarbeiter das Vertrauen in die Führungskräfte verloren haben. Diese instrumentalisierten Whistleblower können sich dann direkt an die übergeordnete Stelle wenden bis zum Vorstand oder Aufsichtsrat (vgl. Conrad, 2016, S. 223 ff.). Ein für die Unternehmen und Aktionäre schädlicher Skandal kann so vermieden werden.

\section{Literatur}

Bass, B.M./Avolio, B.J., Multifactor Leadership Questionnaire, Redwood City 2000.

Brown, M. E./Treviño, L. K., Ethical leadership: A review and future directions, in: The Leadership Quarterly, Vol. 17 (2006), S. 595-616.

$B u ß, E$., Die soziale Kennkarte und Moral der deutschen Top-Manager. Befunde einer empirischen Erhebung. Vortragsmanuskript, Eugen Gutmann Gesellschaft, 2009, 26. März, Online im Internet: URL: www.eugen-gutma nn-gesellschaft.de/upload/vortrag_buss.pdf. (Abrufdatum 28.04.2016). Conrad, C. A., Business Ethics - A Philosophical and Behavioral Approach, Springer International 2018.

Conrad, C. A., Incentives, Risk and Compensation Schemes: Experimental Evidence on the Importance of Risk Adequate Compensation, in: Applied Economics and Finance, Vol. 2 (2015), No. 2, S. 50-55.

Conrad, C. A., Ethische Herausforderungen für die Unternehmensführung, ein Fazit, in: Conrad, Christian, A. (Hrsg.), Unternehmensführung und Ethik. Ansätze zur Erhöhung der Produktivität, Hamburg 2017, S. 233-239. Conrad, C. A., Wirtschaftsethik, - eine Voraussetzung für Produktivität, Berlin, Wiesbaden 2016.

Dahlkamp, S., "In Konzernen machen sich Ego-Typen breit”, in: Spiegel, 28.01.2015, Online im Internet: URL: http://www.spiegel.de/karriere/ka rriere-und-konflikte-psychospielchen-im-management-a-1011453.html (Abrufdatum 28.11.2017).

Göbel, E., Unternehmensethik, Stuttgart 2010.

Information Factory, Schweiz führt?!, Online im Internet: URL: https:// www.information-factory.com/fileadmin/user_upload/studien/Studie_S chweiz_fuehrt_2014.pdf (Abrufdatum 28.11.2017).

Schieffer, A., Führungspersönlichkeit, Struktur, Wirkung und Entwicklung erfolgreicher Top-Führungskräfte, Wiesbaden 1998.

Schwertfeger, B., Wer erfolgreich sein will, muss fies sein, in: Spiegel, 17.10.2016, Online im Internet: URL: http://www.spiegel.de/karriere/ma nager-wer-erfolgreich-sein-will-muss-fies-sein-a-1115117.html (Abrufdatum 28.11.2017).

Staffelbach, B., Management-Ethik, Ansätze und Konzepte aus betriebswirtschaftliche Sicht, Bern, Stuttgart, Wien 1994.

Windolf, P., Korruption, Betrug und "Corporate Governance" in den USA Anmerkungen zu Enron, in: Leviathan Z. Sozialwissenschaft, Vol. 31 (2003), No. 2, S. 185-218. 